\title{
Erratum to: Linear Analysis of Autonomic Activity and Its Correlation with Creatine Kinase-MB in Overt Thyroid Dysfunctions
}

\author{
Manisha Mavai $^{1}$ - Yogendra Raj Singh ${ }^{1}$ - R. C. Gupta ${ }^{2}$ Sandeep K. Mathur ${ }^{3}$. \\ Bharti Bhandari ${ }^{1}(\mathbb{D}$
}

Published online: 4 October 2017

(C) Association of Clinical Biochemists of India 2017

\section{Erratum to: Ind J Clin Biochem \\ DOI 10.1007/s12291-017-0659-0}

The aim of this erratum is to acknowledge the technical error in the online version of the published article (in Table 1).

The corrected Table 1 is given below.

The online version of the original article can be found under doi:10.1007/s12291-017-0659-0.

Bharti Bhandari

drbhartibhandari@yahoo.co.in

1 Department of Physiology, All India Institute of Medical Sciences, Jodhpur, Rajasthan 342005, India

2 Department of Physiology, MGMC, Jaipur, Rajasthan, India

3 Department of Endocrinology, SMS Medical College, Jaipur, Rajasthan, India 
Table 1 Age, BMI and thyroid profile of hypothyroid, hyperthyroid and control groups

\begin{tabular}{lcrrrl}
\hline Parameter $($ Mean $\pm \mathrm{SD})$ & \multicolumn{1}{c}{ Control } & Hypothyroid & Hyperthyroid & $p$ value & Intergroup comparison $p$ value \\
\hline Age $($ years) & $34.49 \pm 10.07$ & $37.3 \pm 9.3$ & $34.37 \pm 8.15$ & 0.34 & $\mathrm{~ns}$ \\
BMI $\left(\mathrm{kg} / \mathrm{m}^{2}\right)$ & $21.29 \pm 1.11$ & $26.16 \pm 3.47$ & $17.47 \pm 1.61$ & 0.000 & $<0.0001 *^{* *}$ \\
Free T3 $(\mathrm{pg} / \mathrm{mL})$ & $2.59 \pm 0.47$ & $1.69 \pm 0.67$ & $9.21 \pm 6.31$ & $<0.001$ & $<0.0001^{\dagger},<0.05^{\ddagger}$ \\
Free T4 $(\mathrm{ng} / \mathrm{dL})$ & $1.29 \pm 0.22$ & $0.70 \pm 0.29$ & $13.57 \pm 6.39$ & $<0.001$ & $<0.0001^{* *}$ \\
TSH $(\mathrm{uIU} / \mathrm{mL})$ & $2.41 \pm 1.45$ & $16.91 \pm 7.38$ & $0.06 \pm 0.05$ & $<0.001$ & $<0.0001 * *$
\end{tabular}

$p<0.05$ were considered statistically significant. BMI body mass index, TSH thyroid stimulating hormone. Intergroup comparison was done using one-way ANOVA followed by post-hoc Bonferroni multiple comparison test (Age, BMI) and Kruskal-Wallis followed by Dunns multiple comparison test (Thyroid profile). * hypo versus hyperthyroids, ${ }^{\dagger}$ hyperthyroid versus control groups, ${ }^{*}$ hypothyroid verses controls, $n s$ non significant 